\title{
Aneurisma da artéria vertebral extracraniana em puérpera portadora de neurofibromatose clássica
}

\author{
Extracranial vertebral artery aneurysm during the puerperium \\ in women with neurofibromatosis type 1 \\ Carla Aparecida Faccio Bosnardo ${ }^{1}$, Rodrigo M achado Landim², \\ Ana Terezinha Guillaumon ${ }^{3}$, M arcus Ageu Baptista ${ }^{4}$, Ricardo H enklain ${ }^{5}$
}

\begin{abstract}
Resumo
Paciente de 26 anos, no 10 으 dia de puerpério, apresentou dor súbita em região cervical esquerda irradiada para região supraclavicular do mesmo lado, seguida de aparecimento de massa pulsátil. Relatava hipertensão sistólica média de 160 mmH g, e diastólica média de 130 $\mathrm{mmH} g$ na gravidez e neurofibromatose. Ao exame físico, apresentava massa pulsátil em região supraclavicular esquerda, sem frêmitos e/ou sopros. U m alargamento do mediastino foi detectado através de raio$x$ de tórax. U ma tomografia computadorizada (região cervical e torácica) evidenciou tumoração em território de artéria subclávia esquerda na sua porção proximal, sem limites definidos pelo extravasamento de contraste, além de hemotórax gigante à esquerda. A paciente evoluiu com choque hipovolêmico, sendo submetida à cirurgia de urgência, com toracotomia seguida de cervicotomia, onde se evidenciou lesão aneurismática rota da artéria vertebral esquerda logo após sua emergência. Após controle do sangramento, procedeu-se à sua ligadura. A paciente evoluiu satiffatoriamente, estando em acompanhamento ambulatorial.
\end{abstract}

Palavras-chave: neurofibromatose, aneurisma, artéria vertebral.

1. Mestre. Médica contratada, Disciplina de Moléstias Vasculares, Departamento de Cirurgia, Hospital das Clínicas, Universidade Estadual de Campinas (UNICAMP), Campinas, SP

2. Residente de $4^{\circ}$ ano, Disciplina de Moléstias Vasculares, Hospital das Clínicas, UNICAMP, Campinas, SP.

3. Doutora. Professora, Disciplina de Moléstias Vasculares, Departamento de Cirurgia, Faculdade de Ciências Médicas, UNICAMP. Chefe do Laboratório de Microprocedimentos e Pesquisas Vasculares, Núcleo de Medicina e Cirurgia Experimental, Faculdade de Ciências Médicas, UNICAMP. Coordenadora do Centro de Referência em Cirurgia Endovascular, UNICAMP, Campinas, SP.

4. Residente de $5^{\circ}$ ano, Disciplina de Moléstias Vasculares, Hospital das Clínicas, UNICAMP, Campinas, SP.

5. Residente de $3^{\circ}$ ano, Disciplina de Moléstias Vasculares, Hospital das Clínicas, UNICAMP, Campinas, SP.

Artigo submetido em 24.01.05, aceito em 20.06.05.

J Vasc Br 2005;4(3):297-300.

Copyright $\odot 2005$ by Sociedade Brasileira de Angiologia e Cirurgia Vascular.

\begin{abstract}
A 26-year-old patient, on the 10th day of the puerperium, presented sudden pain in the left cervical region irradiated to the supraclavicular region on the same side, followed by the presence of a pulsatile mass. She reported a mean systolic hypertension of 160 $\mathrm{mmH} \mathrm{g}$, and mean diastolic hypertension of $130 \mathrm{mmH}$ g in pregnancy and neurofibromatosis. At physical examination, she presented a pulsatile mass in the left supraclavicular region, without thrill and/or murmur. A widening of the mediastinum was detected by a thoracic $X$-ray. A computed tomography (cervical and thoracic region) showed a pulsatile mass in the territory of the left subclavian artery in its proximal portion, without having limits defined by contrast extravasation, besides a giant hemothorax on the left. The patient evolved with hypovolemic shock and was submitted to an urgent surgery, undergoing thoracotomy followed by cervicotomy, in which a ruptured aneurysmal lesion of the left vertebral artery was shown right after its emergency. After controlling the bleeding, a ligation was performed. The patient progressed satisfactorily, having an outpatient follow-up.
\end{abstract}

Key words: neurofibromatosis, aneurysm, vertebral artery.

A neurofibromatose de von Recklinghausen tipo 1 constitui uma alteração genética relacionada ao tecido neuroectodérmico, sendo responsável por $90 \%$ dos casos de neurofibromatose e apresentando incidência de 1:3.000 indivíduos ${ }^{1}$. 0 envolvimento arterial também tem sido descrito, predominantemente acometendo pequenas arteríolas com proliferação intimal, laminações concêntricas eartériasmaiorescom espessamento fibroso intimal. A lesão arterial mais comum é a estenose da artéria renal, levando à hipertensão arterial secundária. A pesar deraros, têm sido descritosaneurismas aorto-ilíacos, viscerais e cervicais. 0 aneurisma da artéria vertebral é de aparecimento raro, principalmente na porção inicial da artéria. A literatura mostra que, 
quando presentes, o local de aparecimento mais comum é a parte intracraniana da artéria.

A neurofibromatoseclássicaégenética, eseussintomas e sinais são evidenciados, de forma geral, na vida adulta ${ }^{1}$.

0 presente relato trata de uma paciente no $100 \mathrm{dia}$ pós-parto, portadoradeneurofibromatoseclássica, com aneurisma roto da artéria vertebral na sua porção extracraniana ${ }^{2,3}$.

\section{Relato do caso}

Paciente de 26 anos, no 10 을 dia de puerpério, apresentou dor súbita em região cervical esquerda irradiada para região supraclavicular do mesmo lado, seguida do aparecimento de tumoração na região há aproximadamente 12 horas. N egava queixas de tontura, síncope ou outros sintomas de isquemia cerebral.

Como antecedentes pessoais, relatava ser portadora de neurofibromatose do tipo 1 e hiperten são na gravidez. Ao exame físico, apresentava-se sem alterações no exame geral, tendo tumoração pulsátil em região supraclavicular esquerda, com cerca de $4 \mathrm{~cm}$ de diâmetro, dolorosa à pal pação e com sopro sistólico. A paciente apresentava manchas café au lait em tronco emembros. 0 raio- $x$ de tórax evidenciou um alargamento de mediastino. A tomografia computadorizada (TC) detórax evidenciou tumoração em território deartéria subclávia esquerda, aneurismanasua segunda porção, sem limites definidos, com extravasamento de contraste(Figura 1), além de hemotórax maciço à esquerda (Figura 2). Ao dar entrada no pronto socorro, a paciente apresentava H B de 8,3 eH T de 26, com pressão arterial (PA) de 80 x $40 \mathrm{mmH}$ g. Ao ser levada à TC, cerca de 1 hora após sua chegada, o H B era de 7,6 e o H T de 21,9. D urante a TC, apresentou taquicardia de 120 bpm ePA de $60 \mathrm{x}$ $20 \mathrm{mmH} \mathrm{g}$, estava descorada e foi levada ao centro cirúrgico de urgência.

D urante o exame, a paciente evoluiu com choque hipovolêmico e foi levada à cirurgia de urgência, sendo submetida à toracotomia esquerda no terceiro espaço para controle proximal, e incisão infraclavicular para controle distal (artéria axilar). Foi feita abordagem supraclavicular da lesão, evidenciando-se hemotórax maciço e aneurisma de primeira porção de artéria vertebral com sangramento ativo, a despeito do pinçamento proximal e distal da subclávia. Foi realizada ligadura proximal da artéria vertebral e, devido ao extenso sangramento retrógrado, foi ampliada a cervicotomia posterior e o controle do sangramento do coto distal dessa artéria. $D$ urante o pinçamento da artéria, 0 coto distal se perdeu, retraindo com cera de osso. Foi colocada entre os processos transversos no local de passagem da artéria vertebral, a fim de obstruir a artéria por compressão.

A paciente evoluiu satisfatoriamenteno pós-operatório, necessitando de nova toracotomia no sexto dia pós-operatório, para retirada de coágulos. Recebeu al ta hospitalar no 24을 dia pós-operatório, mantendo como seqüela cirúrgica o sinal deH orner, queregrediu durante 0 acompanhamento.

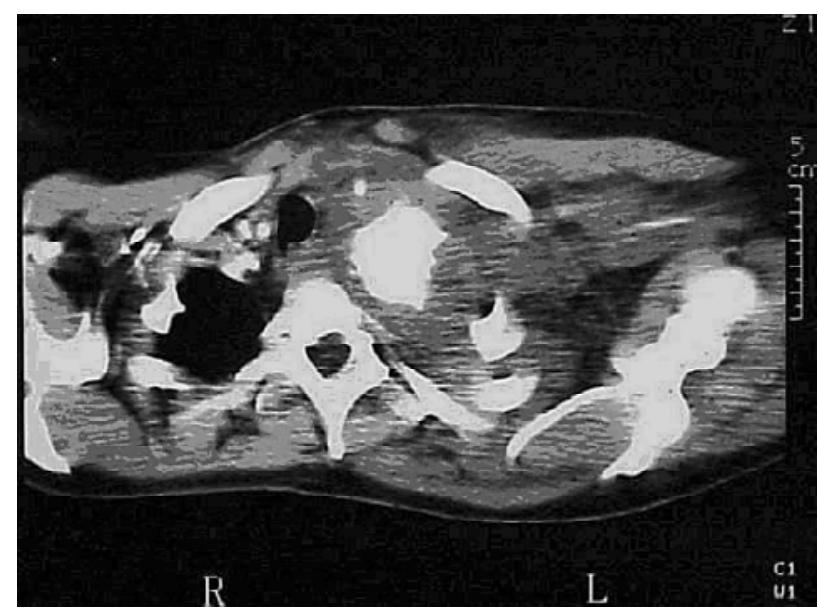

Figura 1 - Tomografia computadorizada evidenciando aneurisma roto de primeira porção de artéria vertebral

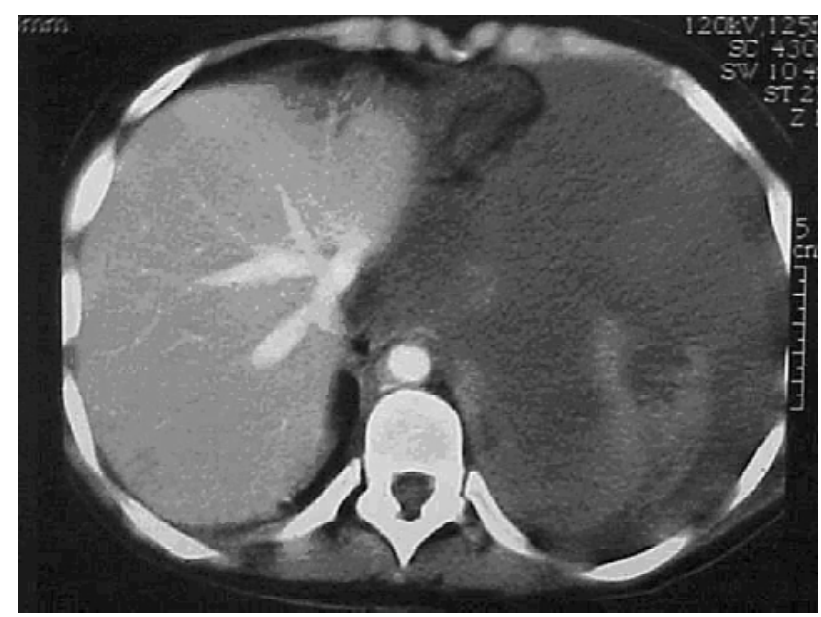

Figura 2 - T omografiacomputadorizada evidenciando he motórax maciço 
Ambulatorialmente, foi submetida à biópsia de nódulo decoxa, queevidenciou neurofibroma cutâneo, à TC, que não evidenciou outros aneurismas na artéria vertebral, e ao duplex scan de carótidas e extremidades, que também não evidenciaram outras anormalidades arteriais. 0 exameanatomopatológico do segmento da artéria vertebral revelou ser sugestivo de aneurisma spurium da artéria.

\section{D iscussão}

A neurofibromatose é uma doença genética que provoca lesões nos sistemas nervoso, digestivo e vascular. No sistema vascular, sua manifestação mais freqüente éa estenose arterial, que pode chegar à oclusão. As principais artérias atingidas são a carótida interna e a renal, levando a seqüel as neurológicas eà hipertensão de difícil controle, respectivamente ${ }^{2,4}$.

A dissecção e a formação de aneurismas não são complicações habituais da neurofibromatose, sendo comumente descritas como componentes de outras síndromes, como Ehlers-D anlos, Klippel-Feil e M arfan. $\mathrm{N}$ a neurofibromatose tipo 1 , as alterações são geralmente intracranianas ${ }^{5}$, sendo a lesão mais prevalente a placa ateromatosa da artéria vertebral em sua origem da artéria subclávia. A incidência de lesões vasculares em pacientes com neurofibromatose tipo 1 tem sido relatada em torno de 3,6\% ${ }^{5}$.

Em 1974, foi sugerido quea proliferação dascélulas de Schwann poderia ser a causa das lesões arteriais na neurofibromatose. $\mathrm{N}$ o mesmo ano, definiram-se duas lesões diferentes, baseadas no tamanho do vaso. $\mathrm{N}$ os vasos maiores, incluindo aorta, carótida e artéria renal proximal, existiria uma invasão direta das células de Schwann e espessamento intimal com destruição da camada média e elástica, levando tanto à estenose como à dilatação. Em vasos menores, a displasia do mesoderma causaria proliferação da musculatura lisa da camada íntima, resultando em estenose e, ocasionalmente, em dilatação pós-estenótica ${ }^{1,6}$.

A paciente relatada neste caso apresentou, durante toda a gestação, hipertensão de difícil controle. Entretanto, os exames de imagem solicitados não evidenciaram lesão anatômica da artéria renal. Assim, a própria gestação poderia ter provocado uma sobrecarga renal, a qual desapareceu após o puerpério6,7.

A presença de hemotórax maciço também tem sido descrita, sendo atribuída ao sangramento de tumores vascularizados de origem mesenquimal, como ganglioneuromas e neurofibromas e sangramento por ruptura de vasos de médio e grande calibres, com enfraquecimento de suas paredes, por exemplo, artéria subclávia ou intercostal ${ }^{8}$. Esta paciente apresentou hematoma inicialmente extrapleural, evoluindo com hemotórax maciço.

A apresentação clínica do aneurisma de artéria vertebral pode estar relacionada à massa da tumoração, a sintomas de origem neurológica, à embolia distal ou, maisraramente, ao choquehipovolêmico, quando ocorre a ruptura do aneurisma, como no caso em questão.

Entre os anos de 1990 e 2003, foram relatados casos esporádicos de lesões aneurismáticas envolvendo as artérias coronária, pancreatoduodenal, poplítea, intercostal, cerebrais extracranianas e cerebrais intracranianas ${ }^{3,5-10}$. N a literatura levantada, não foram encontrados casos semelhantes de aneurisma em artéria vertebral.

0 diagnóstico clínico dos aneurismas arteriais em pacientes portadores de neurofibromatose é, em geral, feito na iminência de complicações como a ruptura ea trombose. A TC éum exame fidedigno para o diagnóstico, por mostrar, além da lesão em si, o envolvimento dos tecidos adjacentes, sendo a ressonância magnética (RM ) necessária nos casos onde existem dúvidas diagnósticas, principalmente no sistema nervoso central. A arteriografia, além de auxiliar no diagnóstico, principalmente nos aneurismas saculares, é necessária no planejamento do tratamento endovascular da doença aneurismática ${ }^{6,8,11,12}$. 0 diagnóstico precoce é possível quando existeinvestigação familiar para a doença ou quando o exame é pedido por outras queixas.

Q uanto ao tratamento, quando o diagnóstico é feito precocemente, só é necessária, muitas vezes, a monitorização do tamanho, além detomografias a cada 6 meses para avaliar o crescimento. É consenso queesses casos devam ser acompanhados por tomografia, sendo que, no caso de crescimento, o procedimento cirúrgico deve ser pensado, embora o crescimento, quando ocorre, seja aparentemente lento.

A técnica endovascular, sempre que possível, deve ser utilizada. N o caso da endovascular não ser possível, a cirurgia aberta com clipagem intracerebral e enxertos pode ser uma alternativa ${ }^{13-17}$.

T odo caso deneurofibromatosedeveser submetido aum check-up (screening) de aneurismas. T odosos casos de neurofibromatose que apresentem antecedentes de hipertensão arterial devem ser exaustivamente pesqui- 
sados inicialmente com duplex arterial, cervical, membros superiores e inferiores, ultra-som abdominal e ecocardiograma. Em caso de suspeita ou dúvida diagnóstica, a TC e ou a RM devem ser solicitadas. Tal procedimento se faz necessário porque os aneurismas são assintomáticos e, quando as roturas existem, o prognóstico ébastantereservado eas seqüel asinúmeras. $\mathrm{N}$ a maioria dos casos observados, o paciente já apresentava hipertensão arterial sistêmica prévia.

\section{Referências}

1. Riccardi VM . von Recklinghausen neurofibromatosis. N Eng J M ed. 1981;305:1617-27.

2. Sasaki J, M iura S, O hishi H, Kikuchi K. N eurofibromatosis associated with multiple intracranial vascular lesions: stenosis of the internal carotid artery and peripheral aneurysms of the H eubner's artery: report case. No Shinkei Geka. 1995;23: 813-17.

3. Zhao JZ, H an XD. Cerebral aneurysm associated with von Recklinghausen'sneurofibromatosis: acasereport. SurgN eurol. 1998;50:592-6.

4. W atano $\mathrm{K}, \mathrm{O}$ kamoto $\mathrm{H}, \mathrm{T}$ akagi $\mathrm{C}, \mathrm{M}$ atsuo $\mathrm{H}, \mathrm{H}$ irao $\mathrm{N}$, Kitabatake A. Neurofibromatosis complicated with $X X X$ syndrome and renovascular hypertension. J Intern Med. 1996;239:531-5.

5. M uhonen M G, GoderskyJC, V angildeJ C. C erebral aneurysms associated with neurofibromatosis. Surg N eurol. 1991;36: 470-5.

6. N akhoul F, Green J, O fer A, Ben-izhak O, Lewin M. Renovascular hypertension associated with neurofibromatosis: two cases and review of the literature. Clin $\mathrm{N}$ ephrol. 2001;55:322-6.

7. Serleth HJ, Cogbill TH, Gundersen SB. Rupture pancreaticoduodenal artery aneurysmsand pheochromocytoma in a pregnant patient with neurofibromatosis. Surgery. 1998;124:100-2.

8. N iimi M, Ikeda Y, Kan S, T akami H, Furui S, T akeshita K. Spontaneous rupture of intercostals artery due to neurofibromatosis type 1 disease treated by percutaneous embolization. Cardiovasc Intervent Radiol. 2002;25:160-1.
9. KirchhofK, V ogt-schaden M , Forsting M . Fusi form aneurysm of the basilar artery in Recklinghausen's disease. R ofo Fortschr G eb Rontgenstr N euen Bildgeb Verfahr. 1996;165:412-14.

10. Tins B, G raves M, Bowling T. N eurofibromatosis associated with coronary artery aneurysm. BrJ Radiol. 2000;73:1219-20.

11. Fabris VE. T umores vasculares. In: Lastória $S, M$ affei $F H A$, YoshidaW B, Rollo H A. D oenças vascularesperiféricas. 3aed. Rio de Janeiro: M edsi; 2002. p. 1798-1799.

12. Faro SH, Mahboubi S, O rtega W. CT diagnosis of rib anomalies, tumors, and infection in children. Clin Imaging. 1993;17:1-7.

13. Villavicencio AT, G ray G, LevequeJ C, FukushimaT, K ureshi $\mathrm{S}$, Friedman $\mathrm{AH}$. U tility of three-dimensional computed tomographic angiography for assessment of relationships between the vertebrobasilar system and cranial base. N eurosurgery. 2001;48:318-27.

14. Johnson CE, Russel EJ, H uckman M S. Resolution of spinal epidural vascular pseudotumor following balloon occlusion of a postoperativevertebral arteriovenousfistula. N euroradiology. 1990;31:529-32.

15. $M$ atsushima $T, M$ atsukado $K, N$ atori $Y$, Inamura $T$, $H$ itotsumatsu $T$, Fukui $M$. Surgery on a sacular vertebral artery-posterior inferior cerebellar artery aneurysm via the transcondylar fossa (supracondylar transjugular tubercle) approach or the trancondylar approach: surgical results and indicationsfor using two different lateral skull baseapproaches. J N eurosurg. 2001;95:268-74.

16. D ayJD , FukushimaT, GiannottaST. C ranial baseapproaches to posterior circulation aneurysms. J Neurosurg. 1997;87: 544-54.

17. Inamasu J, Suga S, Sato S, O nozuka S, Kawase T . Long-term outcome of 17 cases of large-giant posterior fossa aneurysm. Clin N eurol N eurosurg. 2000;102:65-71.

Correspondência:

Carla Aparecida Faccio Bosnardo

Rua V asco Fernandes Coutinho, 555

CEP 13075-253 - Campinas, SP 